\title{
Detection and Restoration of Cracked Digitized Paintings and Manuscripts using Image Processing - A Survey
}

\author{
Nawafil Abdulwahab Farajalla Ali, Imad Fakhri Taha Al Shaikhli \\ Dept of Compute Science, International Islamic University Malaysia, Kuala Lumpur, Malaysia \\ Nawafil.ali@gmail.com \\ Dept of Compute Science, International Islamic University Malaysia, Kuala Lumpur, Malaysia \\ imadf@iium.edu.my
}

\begin{abstract}
The restoration of paintings and manuscripts is defined as the process of restoring old and damaged artworks and documents exhibiting cracks. Cracks are caused by three factors; aging, drying up of painting material, and mechanical. It is necessary that cultural heritages be restored to their original or a near-original state. To enhance the overall quality of the image, there are different techniques and methodologies that can be used for conservation and restoration. The main objective of this study is to analyse techniques and methodologies that have been developed for the detection, classification of small patterns, and restoration of cracks in digitized old painting and manuscripts. The purpose of this research is to present previous works on detection and restoration of cracks using image processing techniques and methodologies.
\end{abstract}

Keywords-Cracks, Detection, Classification, Restoration, Image processing

\section{INTRODUCTION}

Technology has progressed exponentially in the past few decades. Every day, a huge amount of data is generated due to the rapidly increasing size of digital storage space, as well as the advent of the World Wide Web (WWW) [1] Information could contain images or video sequences, as well as synthetic illustration diagrams, charts, or computer aided graphics. Many institutions, such as museums, libraries, galleries, and archives are digitizing their collections to simplify public access. Creating and maintaining the best possible environment for storing or displaying artifacts help prevent damages and increase longevity [2]. It is the prime duty of the custodians of the museums, libraries, galleries, and archives to preserve and conserve their collection, not only in its physical forms, but also digital [3]. Furthermore, digital storage allows the images to be manipulated without risking the original. Collections of paintings saved in museums were from centuries ago, which necessitates protection and restoration so that it remains in good shape indefinitely. Cracking is a common problem in old masters' paintings [4]. A crack appears in paint surfaces when pressures develop within or on it via the action of various factors, causing the material to break[5]. The cracks are mainly caused by aging, drying, and mechanical factors. Age cracks are formed due to non-uniform reduction in the woodpanel support or canvas of the painting, which induce a pressure on the painting's surface[6]. Drying cracks affect the evaporation of the volatile paint's composition and the consequent contraction of the paint. Finally, mechanical actions are caused by painting deformations from external factors, such as projection physical damage impacts . Digital image techniques can be used to develop algorithms that can detect and classify cracks on old digitized paintings and manuscripts[7].

This paper is organized in the following manner. Section II details the detection and restoration techniques. Section III talks about restoration techniques and methods, while section IV discusses detection techniques and methods. The analyses results are presented in section V. Section VI conclude the work and provide some recommendation.

\section{Detection And Restoration TeChNiques}

An easy there are many techniques and methodologies that can be used to detect and restore cracks in digitized old paintings and manuscripts. Selection of specific techniques based to our requirements is crucial. The techniques and methods are categorized into two groups of works. The first deals with restoration, while the second is related to crack detection, as per Fig. (1).

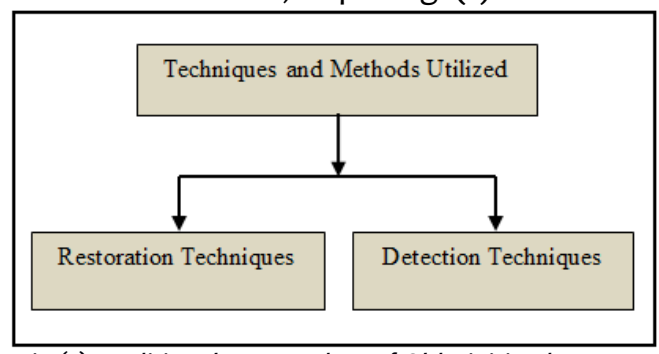

Fig (1) Traditional Approaches of Old Digitized Images 


\section{Restoration Techniques And Methodology}

The restoration of images is a process of recovery of the original appearance of old painting that has been corrupted by natural phenomena and mechanical affects. With the help of image processing techniques, cracks can be detected and the painting restored, which would be beneficial to artwork historian. Various techniques and methodologies can be used for detection and restoration, as follows:

Ioannis Giakoumis et. al 2001[1] proposed an integrated methodology to detect cracks and remove them from old digitized paintings. First, the threshold technique was used to detect cracks on images post-application of the top-hat transform morphology. Thin dark strokes created from artist brush were removed using a semi-automatic procedure based on the region growing method or median radial basis function neural network on hue and saturation data. Finally, the cracks were interpolated using a controlled anisotropic diffusion or order statistics filters. This methodology can be used for the virtual restoration of deteriorated images, and was proven to be quite effective vis-à-vis cultural paintings of high artistic value. The integrated methodology could not detect cracks located on very dark image regions and cracks that cross the border between regions of different colors.

Abas \& Martinez (2002)[8] proposed a methodology of enhancement and detection of cracks in old heritage digital paintings. The multi-oriented Gabor filter operator was used to extract features from the background image of the crack detection phase, which enhances the appearance of cracks from images due to the application of high feature extraction of crack patterns. The result of the proposed methodology confirmed that the Gabor algorithm is a suitable candidate for detecting cracks, and is also scalespecific and multi-orientation detection capable.

Sateesh \& et. al (2003) [4] introduced an integrated approach for crack detection and classification of old deteriorated digitized paintings, of which two main stages of it were implemented in this work. Cracks were detected using the top-hat transform morphology to separate the patterns from the background images, then the thin dark brush strokes, which were misidentified as cracks, are separated using the automatic technique of Median Radial Basic Function (MRBF networks) by applying a semiautomatic approach using the region growth approach. The integrated strategy effective methodology was utilized to virtually restore historical images using semi-automatic approach based on the growth area for crack separation.
Abhilekh \& et. al (2008) [2]proposed a new algorithm for crack detection and restoration of old cultural digitized paintings. The cracks were detected using bottom-hat transformation techniques, followed by the application of the thresholding morphology to accurately detect the cracks. The global thresholding morphology technique operates directly on the bottom-hat transformation. A histogram can be used to introduce binary images. Finally, a new algorithm, called (Modified Adaptive Median Filter) MAMF, was applied to fill in the cracks and patterns in an image. The results of the proposed new algorithm reported a perceived image quality based on the usage of different morphological techniques for detecting more cracks from an image.

Sachin \& et. al (2009) [9]reported a technique that can be used to monitor and interpolate cracks in old heritage digitized paintings. They applied the top-hat transformation algorithm to detect cracks and identify misclassified cracks by separating them from the entire image using a semi-automatic approach based on growth area technique, where a suitable technique modified order statistics was performed. The results of the proposed technique confirmed its viability in identification and separation using different transformation morphologies.

Schirripa \& et. al (2010) [10]proposed a new full automatic approach for crack detection and restoration in historical digitized painting. First, the cracks were detected using the top-hat opening and closing operator transformation method, followed by the separation of brush scratches created from the artist brush being misclassified as cracks on the basis of the Hue and saturation values technique. Finally, the texture synthesis algorithm was used to fill the cracks. The results indicated that the full automatic methodology can efficiently extract the cracks and enhance image quality by filling the cracks.

Shilpa \& Nisha (2012) [7]proposed an algorithm for crack detection and restoration in old historical digitized paintings. They apply a region growing algorithm on the thresholded technique regarded as an output of top-hat transform technique that extract information from the background of an image, and use them to distinguish crack patterns from the strokes of a brush that have been mistakenly identified as cracks. Furthermore, the anisotropic diffusion technique and order statistics filtering were used to fill cracks and restore the digital images. The results confirmed the viability of this method for the classification of crack patterns from images from combining two effective morphology techniques. 
Santhi et. al (2011) [11] proposed an automated detection of pavement crack using the edge detection technique. First, the gray scale morphological operator was used to detect cracks in stored images. Then, a Butterworth filter was used to filter and prepare the image for the next stage, which is the application of the canny edge detection algorithm. Edge-based segmentation technique was utilized to accurately detect the edge. The image was filtered using a Gaussian filter. The image's edge intensity was determined by calculating the gradient in multiple directions from the image's window. Even the brush hairline of a crack can be detected to determine the minimum error rate using edge detection to intensify nonmaximum suppression. Finally, hysteresis was used to eliminate streaking edges that was localized and detected once. The results confirmed the limitations that a given image has to be pre-processed to acquire a grayscale image with the square sized dimensions so that it can be further processed.

Deepika \& Navneet (2013) [12]reported a most popular technique for crack detection and virtual restoration of old deteriorated paintings. They used the top-hat transform technique to detect cracks containing multi-scale morphologies, such as black top hat transformation (BTH), which extract white cracks from black background, and white top hat transformation (WHT), which extract dark cracks from a white background. Both discriminate crack information from the background of an image. Then, the inpainting method was applied for filling or restoring an image. The results confirm the success of the detection methodology to towards obtaining low frequencies.

Shruti \& Sahoo (2014) [13]proposed a technique to detect, restore, and identify cracks in old damaged digitized paintings. A low pass filter was used to detect the cracks. Supervised and unsupervised classification methods were introduced. Four different methods were used to classify cracks from dark black brush strokes before it is filled, which were Back propagation, k-nearest neighborhood, k-mean clustering, and Fuzzy $c$ mean clustering. The results indicated that the fuzzy c-means classifier is the best in the case of many paintings, but it chooses random cluster center initially so that the classes could be interchanged in multiple run. Entropy-based clustering also reported excellent results; it reported lesser brush strokes than fuzzy c, which means that clustering also resulted in lesser number of crack pixels being misclassified as brush strokes.

Sukhjeet \& Amanpeert (2014) [14]posited that the limitations pertaining to crack detection and restoration can be overcome via the development of a new algorithm, which is the nearest neighbor algorithm that can be used to detect and remove cracks. White patterns can be detected and removed by increasing the contrast and saturation technique via the nearest neighbor algorithm. Two measurements were used to compare the results of the nearest neighbor algorithms and the scale invariance highfidelity (SIHF) algorithm, which is a Peak signal to noise ratio (PSNR), and mean squared error (MSE). The results of the proposed algorithm showed that increasing the contrast and saturation techniques improved the images' quality.

Mohammed \& et. al (2014) [15]proposed an automated method for detecting and eliminating cracks in old deterioration digitized paintings. They used the top-hat transform morphology to detect cracks. The Gaussian Classifier technique was used to separate small patterns areas from the cracks. Finally, a new filter called (Modified Trimmed Mean Filter) MTMF and the weighted median filter were used to fill the cracks. The results indicated that the intensity of the crack pixels in these areas were very close to the intensity of the surrounding pixels, rendering this algorithm ineffective in detecting cracks in very dark image region.

Samir (2015) [16]developed an automated crack detection and restoration system for old heritage digitized paintings. The algorithm is composed of two parts; the first was distinguished from the background of the image using the top-tat transformation morphology, which is an algorithm that can improve the subtraction method and morphological operation, while the second was used to identify cracks automatically from image classification using the edge detection technique. The results confirmed the universal applicability of the algorithm vis-à-vis a multitude of images.

Anubhav \& et. al (2015) [17]proposed an integrated algorithm composed of three applications for image processing. The first step was crack detection, where they applied the thresholding technique to generate the morphological top-hat transform to distinguish crack patterns from the background of the image. Then, thin dark strokes of brushes misclassified as cracks were removed using the hit-and-miss cleaning algorithm. Finally, crack filling or restoration was done using median and weighted median filters. The results showed excellent image quality from the usage of different techniques to detect and remove cracks from images and fill the cracks so that the image can be enhanced.

Pratap \& Mandal (2015) [18]introduced an integrated method for detecting and eliminating cracks in old heritage 
digitized paintings. They applied the top-hat and bottomhat transformations based on the structuring element and its shape to probe or interact with a given image. Then, a semi-automatic technique was used to extract the cracks using the hue and saturation technique to separate the thin strokes of the artists' brush from the cracks that was mistakenly identified as cracks. The cracks were then filled using two approaches; inpainting, which is mostly pixelbased, and the pixel-base and patch-based texture synthesis. Both looked for and copied the pixels that were most similar to local neighborhoods as its synthetic texture base. The results showed declination of image quality, which necessitates the usage of apply detection and separation technique to enhance image quality.

\section{Detection Techniques And Methodology}

Nikolaidis \& Pitas (2001) [19]created an algorithm to detect and restore cracks in old digitized paintings. They proposed two main phases: top-hat transformation and thresholding operation, used to detect and eliminate cracks from the background of an image and produce a crack map. The second phase of crack filling was performed using the median filters technique.

Shruti \& Sahoo (2013) [20] reported a system that could be used for cracks detection and restoration in old historical digitized paintings. The thresholding transformation technique was used to identify cracks and classify small patterns from cracks. The semiautomatic classification method was used to distinguish the sizes of the cracks. Four methods were used, supervised by the back propagation algorithm, although it remained unsupervised in fuzzy $c$ mean algorithm. For the filling procedure, different methods were used, such as the modified trimmed mean filter, trimmed mean filter, and alternative mean median filter or variable mean filter. The results confirmed the effectiveness of the methodology of applying different algorithms based on the separation of undesired cracks and thin patterns from images to enhance image quality.

El-Youssef \& Mouhanned (2013) [21]developed a framework that emphasizes geographical analysis of cracks and patterns. For the detection and abstract of the craquelure pattern, three thresholding techniques were applied and compared. Global thresholding, segmented thresholding, and offset thresholding techniques were used to detect and classify small patterns from cracks. The offset method was regarded to be the most accurate technique, with the feature extraction phase being the most important phase. The results of confirmed the effectiveness of the algorithm for crack detection.
Khyati \& Patel (2014) [22] developed an automatic inpainting technique for the detection and restoration of cracks in old historical digitized paintings. Cracks were detected from images based on bottom-hat transform, which is a greyscale morphological algorithm, followed by thresholding transformation morphology to distinguish crack pixels from the background of the image. The cracks were filled using the median algorithm, which function only on local information from the region surrounding the crack pixel, so that quality of content in the other pixels is unaffected. The results showed improved image quality due to the reduction of the whole image blurring being cleared by the median algorithm, which works only on cracked pixel.

Zhe et. al [23] reported a novel algorithm for the detection and restoration of cracks in old heritage digitized painting based on an effective K-means algorithm. The algorithm initially calculated the histogram algorithm in order to determine the initial clustering kernel. An improved K-means algorithm based on the region growing algorithm was then combined. This process was intended to design a new distance function and increase the weight related crack region distance so that we can extract cracks from the background of an image. The algorithm effectively determined the crack information in nonuniform illumination, which resulted in better execution. The results confirmed that the output is not very efficient.

Vidya (2014) [24] proposed an algorithm for crack detection and restoration in old damaged digitized paintings. Cracks were detected and identified using the Gabor technique, which is an integrated methodology for removing scratches and other patterns from images. Cracks were filled with local image information from neighboring pixels, where two methods were used; media and weighted median techniques. The results confirmed the efficiency of the algorithm in detecting major and minor sharpness scratches.

Ram \& Parsai (2015) [25]introduced an algorithm that can remove cracks from images without using the detecting transformation morphology. The nearest neighborhood algorithm used the order of the NL filter. Researchers proposed a new technique for the restoration of cracks alongside pre-processing and post-processing and the nearest neighborhood algorithm. The results showed significant removal of cracks without using top-hat transform, because the nearest neighbor algorithm takes the surrounding pixels to remove cracks from the image painting. Some of these cracks decreased the work of the 
top-hat transformation by directly taking images from specified matrices.

Surish \& et al (2015) [26]presented an automated system to detect and restore cracks from old deteriorated digitized images. Cracks were detected using morphological high pass operator called top-hat transformation to distinguish patterns from its background image. Then, the filling procedure utilized the order statistics algorithm, which deals with the mean and median algorithm. The results showed that the algorithm can be used for a much wider set of applications, due to its accuracy in detecting cracks and thin patterns from images.

Vidya \& Vaibhav (2015) [27] introduced an integrated approach for cracks detection and restoration in old digitized painting. Cracks were detected using the Gabor filter, which controls the sharpness of the pixels. Cracks were filled and restored via the weighted median algorithm, which took into account a nonnegative integer weight at each position in the window size. The results indicated that Gabor features were beneficial towards feature extraction towards the detection of many cracks and scratches. It is also applicable due to its capability for scale-specific and multi-orientation detection.

Balasubramanian et. al [28]proposed a novel approach that combines the top-hat transform morphology and HSV thresholding technique with grey scale thresholding to detect cracks. The degradations of the exterior surface can be measured in civil infrastructures. Crack detection methods can be used to produce gray scale image. The gray scale approach reported excellent results, although they do suffer from the misclassification of edge patterns as cracks. The results showed that HSV thresholding technique was implemented properly, which allowed for the object or element of interest to be extracted. Thereafter, the degradation of the surface can be realized using a simple grey scale thresholding technique, which can be used to classify unaffected areas on the surface degraded regions.

Kaushik \& et. al (2016) [29]introduced a method to develop a semi-automatic crack detection system that analyses the concrete surface and detect cracks from digitized paintings. The crack detection procedure used morphological bottom-hat transformation to separate the pattern from the background of the image. Then, the crack was filled by applying order static median filter based on the information of neighborhood pixels. The results showed significant problem in detecting cracks based on morphological transformation.

\section{RESULT AND DISCUSSION}

There are three main stages that are important towards the detection and restoration of cracks in old historical digitized painting and manuscripts: crack detection, classification of small patterns, and filling.

Researchers proposed mathematical morphological detection of cracks to extract information from the background of the image using various techniques, such as top-hat transform, threshold, bottom-hat, closing operant, segmentation, feature extraction, nearest neighbor, and multiscale morphological $[1,9],[11,14,16,18]$. The crack detection process was applied directly upon the low luminance image, allowing it to detect cracks from the background. However, others suggested the application of different algorithms that are similar to other methods, such as the global threshold, offset method nearest neighborhood, and edge detection [10], [15], [17], [20]. These algorithms reported the most significant and accurate techniques that are capable of detecting the missing areas of cracks and other small damaged areas.

The classification of cracks in deteriorated image was the most important procedure for the enhancement of image quality. Different algorithms were utilized to separate and classify thin dark patterns that could have been detected as cracks based on several techniques, such as Median Radial Basis function (MRBF) neural network, close top-hat operator, semi-automatic technique, supervised, unsupervised, black top-hat, Gaussian classification, region growing, Fuzzy-c, Hue and saturation, and hit and miss cleaning $[2,3,4,5],[7,8,9],[11],[13],[16,17,18,19],[23]$. Distinguishing these thin dark patterns from genuine cracks prior to the implementation of crack filling is necessary to prevent undesirable information. Researchers came up with very effective in algorithms to distinguish small scratches [4], [7], [10, 11], [13], and analyzed how accurate these classifications can be.

Various algorithms were recommended to fill the cracks based on the median trimmed mean filter, close top-hat, median adaptive mean filter, order statistic, anisotropic diffusion, semi-automatic classification, supervised classification, inpainting texture based, median, weighted median, and mean median filter [1], [3], [5- 9] [12], [14, 15], $[18,19],[21-23]$. After identifying cracks and distinguishing the misclassified thin strokes, the researchers restored the cracks using local image information. The authors suggested significant algorithms reported in [13-14], which work well in most stages and produce better output of restoration images. 


\section{CONCLUSION AND FUTURE WORK}

Image detection and restoration is very significant research area of image processing. Image restoration is concerned with recovering an original image damaged by different forms of degradation. In this paper, different techniques and methodologies were reviewed on detection, classification, and restoration of cracks in old digitized images and they were compared on the bases of their utilization and advantages. In the future, researchers can analyse and implement steps in the empirical usage of software to develop and modify a full automated algorithm to detect and remove cracks and improve and develop algorithms that can be used to extract information to accurately classify patterns and shapes.

\section{REFERENCES}

[1] N. Nikolaidis and I. Pitas, "Digital image processing in painting restoration and archiving," in Image Processing, 2001. Proceedings. 2001 International Conference on, 2001, pp. 586589.

[2] I. Giakoumis, N. Nikolaidis, and I. Pitas, "Digital image processing techniques for the detection and removal of cracks in digitized paintings," IEEE Transactions on Image Processing, vol. 15, pp. 178-188, 2006.

[3] F. Abas and K. Martinez, "Craquelure analysis for content-based retrieval," in Digital Signal Processing, 2002. DSP 2002. 2002 14th International Conference on, 2002, pp. 111-114.

[4] M. Barni, A. Pelagotti, and A. Piva, "Image processing for the analysis and conservation of paintings: opportunities and challenges," IEEE Signal processing magazine, vol. 22, pp. 141144, 2005.

[5] D. S. R. Y. Sateesh.V1, "IMPLEMENTATION OF DETECTION AND REMOVAL OF CRACKS IN DIGITIZED PAINTINGS USING DIP TECHNIQUES," Global Journal of Advanced Engineering Technologies, 2012.

[6] V. K. Abhilekh Gupta, Abhinav Gupta and M. C. Srivastava, "Image Processing Methods for the Restoration of Digitized Paintings," Thammasat Int. J. Sc. Tec, vol. 13, p. 7, 2008.

[7] S. A. N. SHARMA2, "INPAINTING APPROACH TO REPAIR CRACKED IMAGES " International Journal of Application or Innovation in Engineering \& Management vol. 1, p. 7, 2012.

[8] F. S. A. a. K. Martinez, "CRAQUELURE ANALYSIS FOR CONTENT-BASED RETRIEVAL," p. 5, 2002.

[9] S. V. S. a. M. A. R. Mahajan2, "Cracks Inspection and Interpolation in Digitized Artistic Picture using Image Processing Approach " International Journal of Recent Trends in Engineering, vol. 1, p. 3, 2009.

[10] F. S. G Schirripa Spagnolo1, "Virtual restoration of cracks in digitized image of paintings " International Conference on Defects in Insulating Materials, p. 8, 2010.

[11] b. Qin Zoua, c,爪, Yu Caoc, Qingquan Lib,d, Qingzhou Maob,d, Song Wangc "Automatic crack detection from pavement images," p. $11,2011$.

[12] N. K. Deepika Pagrotra1, "A Review Paper on Crack Detection and Restoration of Old Painting," International Journal of Science and Research vol. 4, p. 3, 2015.

[13] G. S. Shruti Garg1, "Classification of Cracks and Brush Strokes in Old Digital Paintings " Int. J. of Recent Trends in Engineering \& Technology, , vol. 11, p. 10, 2014

[14] A. K. Sukhjeet Kaur, "Sukhjeet Kaur, Amanpeert Kaur " International Journal Of Engineering And Computer Science vol. 3 , p. 6 .

[15] N. A. W. F. P. Mohammed AbdALLA A. ElmaleehP1P, Amin Babikr A/Nabi MustafaP2 "Detection and Removal of Cracks in
Digitized Paintings via Digital Image Processing " International Journal of Innovative Science, Engineering \& Technology, vol. 1, p. $8,2014$.

[16] S. K. Bandyopadhyay, "Crack Detection and Classification Based on New Edge Detection Method " Bandyopadhyay, International Journal of Advances in Computer Science and Technology, vol. 4 p. 5.

[17] A. K. B. Anubhav Singh1, Ekta Tamrakar3 "Crack Filling of Art by using Image Processing: A Review " INTERNATIONAL JOURNAL OF INNOVATIVE RESEARCH IN ELECTRICAL, ELECTRONICS, INSTRUMENTATION AND CONTROL ENGINEERING vol. 3, p. 5 .

[18] P. C. Mandal, "A Survey of Detection and Removal of Crack from Digital Painting " International Journal of Advanced Research in Computer Science and Software Engineering vol. 5, p. 4

[19] I. G. a. I. Pitas, "DIGITAL RESTORATION OF PAINTING CRACKS " IEEE Signal processing magazine, p. 4, 2001.

[20] G. S. Shruti Garg1, "A Comparative Study of Classification Methods for Cracks in Old Digital Paintings " Proc. of Int. Conf. on Emerging Trends in Engineering and Technology p. 6, 2013.

[21] M. El-Youssef, "The extraction and classification of craquelure patterns for geographical analysis of fine art painting " p. 27, 2013.

[22] K. T. V. a. N. M. Patel, "Automatic Crack Detection and Inpainting " International Journal of Computer Science Engineering vol. 3, p. 5.

[23] L. Z. Cui Fang 1, and Yao Li 3 "Images Crack Detection Technology based on Improved K-means Algorithm " JOURNAL OF MULTIMEDIA, vol. 9, p. 7, 2014

[24] V. V. Khandare, "Detection \& Removal of Cracks in Digitized Paintings Based on Image Processing " International Journal of Engineering Research \& Technology vol. 3, p. 5, 2014.

[25] R. K. G. P. M. P. Parsai2, "Restoration of Digitized Image of Cracked Paintings -A Review "International Journal for Scientific Research \& Development, vol. 3, p. 3, 2015.

[26] R. B. a. P. S. Soji Koshy "Strength Analysis of Buildings using Image Processing and SHM Principles " International Conference on Emerging Technological Trends p. 6, 2016.

[27] V. V. K. Vidya V. Khandare1, "Digital Restoration of Cracks Based on Image Processing "International Research Journal of Engineering and Technology vol. 2, p. 4, 2015.

[28] E. B. S. Sankarasrinivasana, *, K. Karthika, U. Chandrasekarb and Rishi Guptac "Health Monitoring of Civil Structures with Integrated UAV and Image Processing System," Procedia Computer Science p. 8, 2015.

[29] K. B. a. S. K. Bandyopadhyay, "DIGITAL IMAGE PROCESSING TECHNIQUES FOR THE DETECTION AND REMOVAL OF CRACKS IN DIGITIZED PAINTINGS OR CONCRETE RTRUCTURE," International Journal of Current Research, vol. 8, p. 6, 2016 